\title{
Sistema de Compensação de Controlador PID via Redes Neurais Artificiais para Altitude de Quadrimotor
}

\author{
Daniel Trovão Simões*. João Viana da Fonseca Neto **. Anselmo Cardoso de Paiva*** \\ *Universidade Federal do Maranhão, São Luís, MA 65000-000 \\ Brasil (e-mail: eng.daniel.trovao@gmail.com). \\ ** Universidade Federal do Maranhão, São Luís, MA 65000-000 \\ Brasil(e-mail: jviana@dee.ufma.br) \\ *** Universidade Federal do Maranhão, São Luís, MA 65000-000 \\ Brasil(e-mail:paiva@nca.ufma.br)
}

\begin{abstract}
The PID controller compensation system via artificial neural networks for quadrotor altitude is presented in this paper in order to improve the tuning of height controllers in quadricopters. Because it is a second-order system, the altitude control of these model airplanes is most often done using a proportional and derivative controller (PD). This control strategy presents good results; however low or even lack of adaptability. This article presents a neural compensator consisting of a neural PID controller and a gain adjustment network in order to guarantee the adaptability to the PD controller for parametric variations and a better alignment of proportional and derivative gains.
\end{abstract}

Resumo: O sistema de compensação de controlador PID via redes neurais artificiais para altitude de quadrimotor é apresentado neste artigo com o intuito de melhorar a sintonia dos controladores de altura em quadricópteros. Por se tratar de um sistema de segunda ordem, o controle de altitude destes aeromodelos é feito na maioria das vezes utilizando um controlador proporcional e derivativo (PD). Este controle apresenta bons resultados, porém com baixa ou até ausência adaptabilidade. Neste Artigo apresenta-se um compensador neural constituído de um controlador PID neural e uma rede de ajuste de ganhos a fim de garantir à adaptabilidade ao controlador PD para variações paramétricas e uma melhor sintonia dos ganhos proporcional e derivativo.

Keywords: Neural Networks; PID Control; Delta Rule; Hebbian learning; Quadrimotors.

Palavras-chaves: Redes Neurais; Controlador PID; Regra Delta; Aprendizado Hebbiano; Quadrimotores.

\section{INTRODUÇÃO}

Para muitos problemas de controle, principalmente aqueles relacionados a processos industriais (Fonseca Neto, et al., 2018), o controlador Proporcional, Integral e Derivativo (PID) é a principal solução de controle utilizada. A estrutura do controlador é relativamente simples e três parâmetros para ajuste. Fazendo com que seja amplamente utilizado em qualquer sistema de acionamento (Yibo \& Shuxi, 2012). Além disso, os controladores PID clássico apresentam baixo custo computacional e baixo consumo energético (Moura, et al., 2018). Contudo, O PID clássico apresenta baixa adaptabilidade, pois seus parâmetros são fixos constantes (Necdet, et al., 2015).

Com a evolução de técnicas computacionais vieram do advento das redes neurais artificiais (RNA) (McCulloch e Pitts, 1943) que são sistemas de processamento de informação nãolineares adaptativos que combinam numerosas unidades de processamento com uma série de características como auto adaptação, auto-organização e aprendizado em tempo real
(Shifei \& Hui, 2011). Durante a década de 80 a pesquisa sobre as RNAs tem feito desenvolvimentos notáveis, e as elas têm sido amplamente aplicadas (Haykin, 2008). Sendo uma boa alternativa considerar nos projetos de controles PID a componente de adaptabilidade.

As redes neurais tem a habilidade de aprender funções matemáticas. A importância desta auto-aprendizagem é essencial em muitos métodos tradicionais de controle adaptativo e ótimo (Jinkun, 2017).

Com grandes avanços em veículos aéreos não tripulados, um tipo de configuração que tem atraído a atenção de vários pesquisadores, é o quadrimotor. Estes sistemas de múltiplos rotores são capazes de pairar e possuem boas capacidades de manobra. Do ponto de vista mecânico, eles podem ser considerados mais simples que os helicópteros, porque a disposição dos quatro motores elimina os torques giroscópios excluindo a necessidade de um motor de cauda. (Rong \& Umit, 2006) 
Uma das primeiras abordagens de controle de quadricópteros foi o controlador PID clássico que é relativamente simples (Moura, et al., 2018). Este controlador tem uma boa robustez para a rejeição à perturbação e os parâmetros são fáceis de ajustar, pois há somente três fatores de sintonia. Isto leva a ser amplamente utilizado no sistema de acionamento total. Contudo, o PID clássico é muito adequado para este tipo de aeromodelo, que possui características de não-linearidades, controle sub-atuado e forte acoplamento das entradas. (Jun \& Yuntang, 2011) (Bresciani, 2008)

O objetivo deste artigo é apresentar um compensador neural (Controlador PID Neural e rede de ajuste de ganhos) com o intuito de compensar a não linearidade de modelos em quadrimotores.

Este artigo é organizado em quatro seções: a Seção 2, descrição operacional da planta retrata o sistema utilizado para desenvolvimento deste trabalho, o modelo de altitude de um quadrimotor. Na Seção 3 é apresentada a descrição do controlador PD (proporcional e derivativo), controlador PID RNA e a rede de ajuste de ganhos. Resultados e simulações são mostrados com as variáveis que são necessárias para executar os algoritmos, como também discussões comparativas na Seção 4. Finalmente, a conclusão do artigo é apresentada na Seção 5 e explicitando se houve melhorias ou não no desempenho do controlador.

\section{DESCRIÇÃO OPERACIONAL DA PLANTA}

Quadricópteros estão em rápida ascenção no campo da robótica e ganharam popularidade em aplicações comerciais, tais como: filmagens e fotografias aéreas. Eles são capazes de levar cargas e possuem uma performance de voo que permite suportar uma série de aplicações em ambiente abertos e confinados (Vijay, et al., 2012). O funcionamento dos quadrimotores é baseado na variação das velocidades angulares dos quatro motores acoplados cada um localizado em cada extremidade no veículo. Com a variação angular dos motores, este veículo é capaz de realizar movimentos angulares (Rolagem, Arfagem e Guinada) como também movimentos translacionais nos eixos (X, Y e Z), conforme ilustrado na Fig. 1 (Bresciani, 2008).

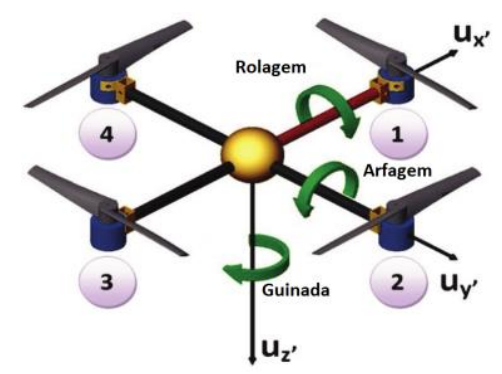

Fig. 1: Movimentos do Quadrimotor.

\subsection{Modelo Matemático do Atuador e Processo}

Com quatro motores, que são quatro entradas de controle, e seis movimentos de saída (graus de liberdade): três rotacionais e três translacionais. Portanto, trata de um sistema suba- tuado, pois o número de entradas é menor que o de saídas. Como também a planta discutida neste artigo, trata de uma modelo de múltiplas entradas e múltiplas saídas (MIMO). Isto oferece um desafio a mais para o controle deste modelo. As equações das entradas, (Bouadallar, et al., 2006), desse sistema são dadas por

$$
\begin{aligned}
& U_{1}=b\left(\Omega_{1}^{2}+\Omega_{2}^{2}+\Omega_{3}^{2}+\Omega_{4}^{2}\right), \\
& U_{2}=b\left(\Omega_{4}^{2}-\Omega_{2}^{2}\right), \\
& U_{3}=b\left(\Omega_{3}^{2}-\Omega_{1}^{2}\right), \\
& U_{4}=d\left(-\Omega_{1}^{2}+\Omega_{2}^{2}-\Omega_{3}^{2}+\Omega_{4}^{2}\right),
\end{aligned}
$$

sendo $U_{1}, U_{2}, U_{3} e U_{4}$, as entradas a força empuxo no eixo $\mathrm{Z}$ e os torques nos eixos $\mathrm{X}, \mathrm{Y}$ e $\mathrm{Z}$. Assim como, $\Omega_{1}^{2}, \Omega_{2}^{2}, \Omega_{3}^{2} e \Omega_{4}^{2}$ são as velocidades angulares em cada motor variando de 1 a 4 . As variáveis $b$ e $d$ são responsáveis por converter velocidade angular em força e toque, respectivamente.

O modelo completo da dinâmica do quadrimotor para os movimentos em $\mathrm{X}$, Y e Z e consequentemente os movimentos de arfagem, rolagem e guinada são dados por

$$
\begin{aligned}
& \ddot{x}=(\cos \phi \sin \theta \cos \psi+\sin \phi \sin \psi) \frac{1}{m} U_{1}, \\
& \ddot{y}=(\cos \phi \sin \theta \sin \psi+\sin \phi \cos \psi) \frac{1}{m} U_{1}, \\
& \ddot{z}=-g+(\cos \phi \cos \theta) \frac{U_{1}}{m}, \\
& \ddot{\phi}=\dot{\theta} \dot{\psi}\left(\frac{I_{y}-I_{z}}{I_{x}}\right)+\frac{U_{2}}{I_{x}}, \\
& \ddot{\theta}=\dot{\phi} \dot{\psi}\left(\frac{I_{z}-I_{x}}{I_{y}}\right)+\frac{U_{3}}{I_{y}}, \\
& \ddot{\psi}=\dot{\phi} \dot{\theta}\left(\frac{I_{x}-I_{y}}{I_{z}}\right)+\frac{U_{4}}{I_{z}},
\end{aligned}
$$

sendo $I_{x}, I_{y} e I_{z}$ são os momentos inerciais, $\phi, \theta, e \psi$ são os ângulos de arfagem, rolagem e guinada. $m$ a massa do quadrimotor e $g$ a gravidade.

O sistema de equações diferenciais possui diversas variáveis de controle e de saída, bem como constantes que regem a dinâmica do sistema. Isto torna o sistema de controle bastante complexo.

\subsection{Simplificação Modelo Quadrimotor}

A fim de transformar o conjunto de equações que é um sistema MIMO em um sistema mais simples SISO. O artigo foca no controle da altitude que podem ser representadas pela dinâmica, (Bouadallar, et al., 2006), de entrada dada por 


$$
U_{1}=b\left(\Omega_{1}^{2}+\Omega_{2}^{2}+\Omega_{3}^{2}+\Omega_{4}^{2}\right),
$$

Como também, a equação de aceleração no eixo $\mathrm{Z}$ dado por

$$
\ddot{z}=-g+(\cos \phi \cos \theta) \frac{1}{m} U_{1},
$$

As Eq.(3) e Eq.(4) são as equações para o controle da dinâmica altitude do quadrimotor e são utilizadas para colocá-lo em determinada altura.

\section{FORMULAÇÃO DO PROBLEMA}

Nesta seção a estrutura dos controladores PD, o controlador PID neural, a rede de ajuste de ganhos e os algoritmos de aprendizados das redes neurais são mostrados e explicados através de equações matemática e a representação em diagrama de blocos.

A estrutura geral de controle da Fig.2 é composta pelo controlador $\mathrm{PD}\left(U_{P D}\right)$ em paralelo com o neural $\left(U_{N}\right)$, na qual os sinais de ambos os controladores são somados e o esforço de controle $(U)$ é enviado para a planta. Este sinal de esforço é comparado com a saída de uma outra rede neural $\left(U_{I N}\right)$ e erro resultante $\left(e_{I N}\right)$ desta comparação é utilizado para ajustar os pesos desta estrutura. Esta rede é denominada de rede de ajuste de ganhos neural (Liu, 2016).

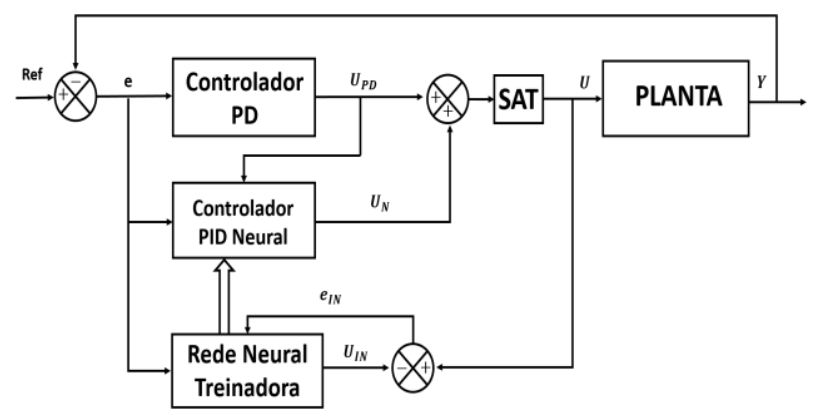

Fig. 2: Estrutura do Compensador PD via Rede Neural.

Na proposta deste artigo a estrutura do compensador é modificada. Um bloco de saturação denominado por (SAT) na Fig.2 é adicionado a planta, pois em aplicações reais, o sinal de controle é limitado por questões físicas do processo.

\subsection{Estrutura do Controlador PD para Quadrimotor}

A estrutura do controlador PD utilizada para o controle do eixo $\mathrm{Z}$ é dada pela Eq.(5). Esta configuração de controle é amplamente utilizada para controle de altitude de quadrimotores, (Quan, 2017) (MORADI \& JOHNSON, 2005), que é dado por

$$
U_{P D}(k)=U_{P D}(k-1)+K_{P} e(k)-K_{D} \dot{e}(k)
$$

e tem a estrutura de blocos ilustrada na Fig.3.

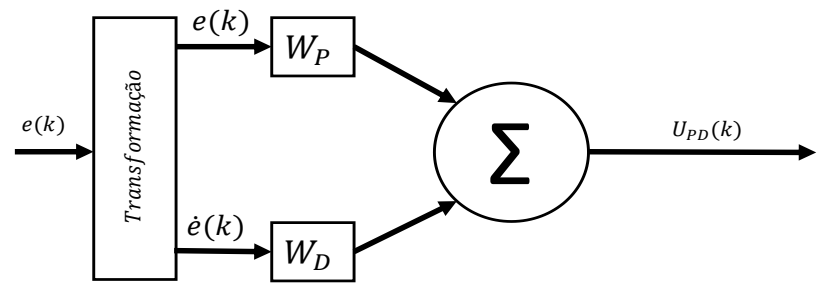

Fig. 3: Controlador PD.

\subsection{Estrutura do Controlador PID neural e Rede de Ajuste de Ganhos.}

A configuração do controlador neural PID e rede de ajuste de ganhos são compostos pelas componentes proporcionais, integrativas e derivativas que são os pesos sinápticos da rede neural $\left(w_{i}^{N}\right)$ e com os elementos de entrada da rede $\left(x_{i}^{N}\right)$ que são o erro com seus elementos integrativos e derivativos, (Liu, 2016), é dada por

$$
U_{N}(k)=U_{N}(k-1)+\frac{K_{s}^{N} \sum_{i=1}^{3} x_{i}^{N}(k) w_{i}^{N}(k)}{\sum_{i=1}^{3}\left|w_{i}^{N}(k)\right|} .
$$

A rede neural que identifica o controlador PID neural possuem a mesma estrutura com o mesmo vetor de entrada e a normalização dos pesos em ambas as redes evitam com que os sinais das redes não cresçam de forma exponencial (Xiwen, 2008) (Jinkun, 2017).

Os elementos de entrada das redes neurais são dados por

$$
\begin{aligned}
& x_{1}(k)=e(k)-e(k-1), \\
& x_{2}(k)=e(k) * T s, \\
& x_{3}(k)=(e(k)-2 e(k-1)+e(k-2)) / T s,
\end{aligned}
$$

sendo $x_{1}$ é o erro, $x_{2}$ é a integral do erro e $x_{3}$ é derivada do erro (Liu, 2017). A estrutura de blocos da Eq.(6) é ilustrada na Fig.4.

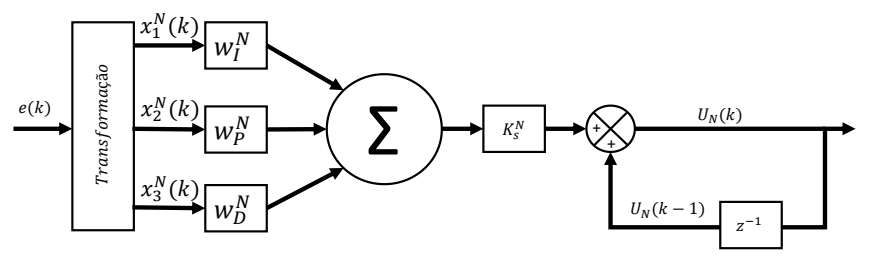

Fig. 4: Estrutura do Controlador Neural e Rede de ajuste de ganhos.

Ambas as redes possuem o ajuste do ganho $K_{s}^{N}$. Este ganho é chamado de coeficiente de proporção e sua escolha é importante. Com um valor alto de $K_{s}^{N}$, a resposta de controle do sistema é melhor, porém isto causa um grande sobressinal que poderá levar o sistema a instabilidade. Portanto, deve ser 
escolhido de forma adequada o valor deste ganho (Xiwen, 2008).

\subsection{Algoritmos de Atualização dos Ganhos}

De acordo com a teoria de rede neurais. Há diversos algoritmos de aprendizagem para atualização dos pesos sinápticos (Haykin, 2008). Nesta seção são apresentados os algoritmos Hebbiano supervisionado e não supervisionados assim como a regra delta.

\subsubsection{Algoritmo de Ajuste de Pesos de Redes Neurais}

As diferentes formas de aprendizado são mostradas nas próximas equações. Onde $\eta_{P}, \eta_{I}$ e $\eta_{D}$ são as taxas de aprendizados dos ganhos proporcionais, integrativos e derivativos do sinal de erro e $w_{i}$ são os pesos da rede. As regras são dadas por

1) Regra de aprendizado (Hebbiano não supervisionado):

$$
\begin{aligned}
& w_{P}(k)=w_{P}(k-1)+\eta_{P} x_{1}(k-1) u(k-1) \\
& w_{I}(k)=w_{I}(k-1)+\eta_{I} x_{2}(k-1) u(k-1) \\
& w_{D}(k)=w_{D}(k-1)+\eta_{D} x_{3}(k-1) u(k-1)
\end{aligned}
$$

2) Regra de aprendizado (Delta supervisionado):

$$
\begin{aligned}
& w_{P}(k)=w_{P}(k-1)+\eta_{P} e(k-1) x_{1}(k-1) \\
& w_{I}(k)=w_{I}(k-1)+\eta_{I} e(k-1) x_{2}(k-1) \\
& w_{D}(k)=w_{D}(k-1)+\eta_{D} e(k-1) x_{3}(k-1)
\end{aligned}
$$

3) Rede de aprendizado (Hebbiano supervisionado):

$$
\begin{aligned}
& w_{P}(k)=w_{P}(k-1)+\eta_{P} e(k-1) x_{1}(k-1) u(k-1) \\
& w_{I}(k)=w_{I}(k-1)+\eta_{I} e(k-1) x_{2}(k-1) u(k-1) \\
& w_{D}(k)=w_{D}(k-1)+\eta_{D} e(k-1) x_{3}(k-1) u(k-1)
\end{aligned}
$$

\subsubsection{Algoritmo da Rede de Ajuste dos ganhos do Compen- sador PID Neural}

Para treinamento dos pesos da rede de ajuste dos ganhos é utilizada a regra delta supervisionada, pois esta evita com que os pesos sinápticos tendam para valores muito altos e o sinal de erro é dado por

$$
e(k)_{I N}=U(k)-U_{I N}(k),
$$

sendo $(U)$ o sinal de controle da planta e $\left(U_{I N}\right)$ o sinal da rede de ajuste de pesos.

A regra de aprendizado delta foi estabelecida da seguinte forma dado por:

$$
\begin{aligned}
& w_{P}^{I N}(k)=w_{P}^{I N}(k-1)+\eta_{P}^{I N} e(k-1)_{I N} x_{1}(k-1) \\
& w_{I}^{I N}(k)=w_{I}^{I N}(k-1)+\eta_{I}^{I N} e(k-1)_{I N} x_{2}(k-1) \\
& w_{D}^{I N}(k)=w_{D}^{I N}(k-1)+\eta_{D}^{I N} e(k-1)_{I N} x_{3}(k-1)
\end{aligned}
$$

sendo $\eta_{P}^{I N}, \eta_{I}^{I N}, \eta_{D}^{I N}$ as taxas de aprendizagem dos ganhos proporcional, integrativo e derivativo da rede de ajuste de ganhos.

\subsubsection{Algoritmos de Ajuste de Pesos do Controlador PID Neural}

Os ganhos da rede Controlador PID foi também utilizado a regra delta supervisionada. Neste caso, o sinal que supervisiona a atualização dos ganhos é dado por

$$
e(k)_{N}=U_{P D}(k) .
$$

A regra delta utilizada no controlador PID neural é a mesma da rede de ajuste de ganhos, porém a diferença está no peso atual do treinamento, (Liu, 2016), com a seguinte equação dada por

$$
\begin{aligned}
& w_{P}^{N}(k)=w_{P}^{I N}(k)+\eta_{P}^{N} e(k-1)_{N} x_{1}(k-1) \\
& w_{I}^{N}(k)=w_{I}^{I N}(k)+\eta_{I}^{N} e(k-1)_{N} x_{2}(k-1) \\
& w_{D}^{N}(k)=w_{D}^{I N}(k)+\eta_{D}^{N} e(k-1)_{N} x_{3}(k-1),
\end{aligned}
$$

sendo $\eta_{P}^{N}, \eta_{I}^{N}, \eta_{D}^{N}$ as taxas de aprendizagem dos ganhos proporcional, integrativo e derivativo do controlador PID neural.

\section{EXPERIMENTOS COMPUTACIONAIS}

A fim de avaliar a eficácia do método de compensação com o controlador PID neural. Nesta seção são apresentadas simulações e comparações com os resultados do método tradicional de controle. Nos dois sistemas separados, usamos o mesmo modelo e controlador PID discreto.

Com as Eq.(3) e Eq.(4) do modelo e as Eq.(6), Eq.(11) e Eq.(13) que são a equação de entrada e a equação do modelo assim como as estruturas das redes neurais (controladora e ajuste de ganhos e por fim a Eq.(13) que é o algoritmo de regulação de ganhos das redes.

Para enfatizar as qualidades da proposta em relação aos controladores existentes, o sistema também é simulado com o controlador PID tradicional. Como dito anteriormente, Em seguida, as saídas dos dois sistemas e o sinal do passo original são todos mostrados no mesmo gráfico.

O quadricóptero utilizado para os experimentos computacionais é o Rolling Spider, por tratar de um modelo que pode ser facilmente acessado pelo software MATLAB na versão 2018 com Toolbox Aerospace Blockset. Os parâmetros deste artigo 
são extraídos do modelo matemático que regem o funcionamento deste quadrimotor.

Todas as variáveis utilizadas na simulação são sumarizadas na Tabela 1 (MATHWORKS, 2018).

\section{Tabela 1. Parâmetros de Simulação}

\begin{tabular}{l|c|c}
\hline \multicolumn{1}{c|}{ Variável } & Unidade & Valor \\
\hline Passo de Simulação(Ts) & {$[\mathrm{s}]$} & 0,005 \\
\hline Gravidade(g) & {$\left[\mathrm{ms}^{2}\right]$} & 9,8 \\
\hline Massa(m) & {$[\mathrm{kg}]$} & 0,08 \\
\hline Coeficiente de força (b) & {$\left[\mathrm{Ns}^{2}\right]$} & $4,72 \mathrm{e}-8$ \\
\hline Saturação Maxíma (Smax) & {$[\mathrm{N}]$} & 1,2272 \\
\hline Saturação Mínima (Smin) & {$[\mathrm{N}]$} & 0,0245 \\
\hline Arfagem $(\phi)$ & {$\left[{ }^{\circ}\right]$} & 0 \\
\hline Rolagem $(\theta)$ & {$\left[^{\circ}\right]$} & 0 \\
\hline Kp & {[]} & 0,8 \\
\hline Kd & {[]} & 0,3 \\
\hline Com
\end{tabular}

Com os variáveis de controle das Eq.(3) e Eq.(4), o próximo passo é desenvolver os algoritmos PID RNA e a rede de ajuste de ganhos. Os parâmetros de inicialização para estes algoritmos são apresentados na Tabela 2.

Tabela 2. Valores do Controlador PID Neural e Rede Neural de treinamento

\begin{tabular}{l|c}
\hline \multicolumn{1}{c|}{ Variável } & Valor \\
\hline$\eta_{I}^{N}, \eta_{P}^{N}, \eta_{D}^{N}$ & 0,$45 ; 0,55 ; 0,15$ \\
\hline$w_{I}^{N}, w_{P}^{N}, w_{D}^{N}$ & $1 ; 5 ; 0,5$ \\
\hline$K_{s}^{N}$ & 0,07 \\
\hline$\eta_{I}^{N}, \eta_{P}^{N}, \eta_{D}^{N}$ & 0,$315 ; 0,12 ; 0,054$ \\
\hline$w_{I}^{N}, w_{P}^{N}, w_{D}^{N}$ & 0,$001 ; 0,01 ; 0,01$ \\
\hline$K_{s}^{N}$ & 1,25 \\
\hline
\end{tabular}

Após inicializado as variáveis das redes e de simulação ambos os controladores foram submetidos à resposta ao degrau e comparado os desempenhos.

\subsection{Resposta dos Controladores ao Degrau}

A resposta ao degrau é utilizada para análise comparativa mais utilizados para avaliar o desempenho dos controladores. $\mathrm{Na}$ Fig. 5 é possível observar a resposta ao degrau do controlador PD e controlador PD com a rede neural.

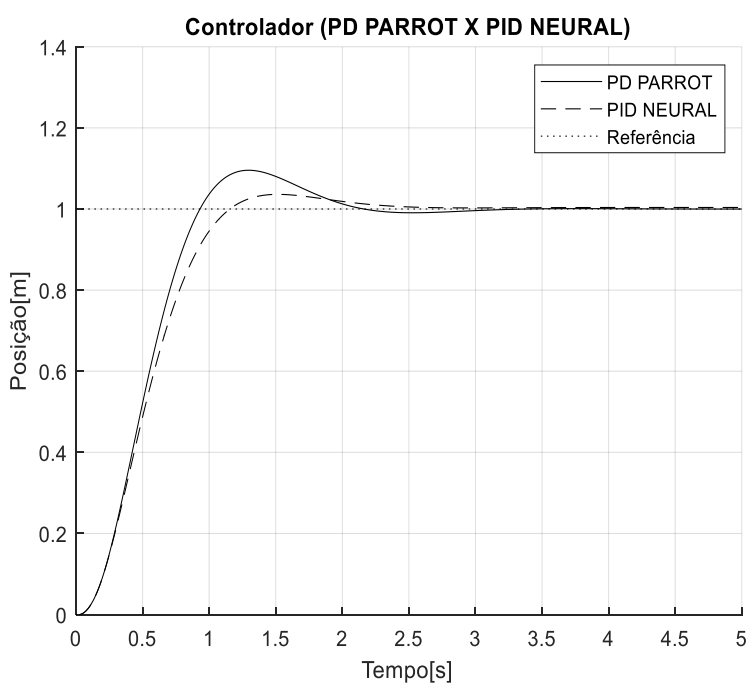

Fig. 5: Resposta do Controladores.

O controlador PID neural, em linha tracejada, apresentou uma resposta melhor ao degrau unitário no quesito sobressinal e tempo de acomodação que o sistema PD tradicional. Contudo, o tempo de subida é maior para PID neural em comparação ao PD Parrot.

\subsection{Análise dos Estados do Modelo}

A outra maneira avaliativa de análise são os próprios estados da planta e como o sistema trata da aceleração do quadrimotor no eixo $\mathrm{Z}$ visto em Eq.(4). Os estados são posição e velocidade. Esses estados são avaliados pelo controlador PD tradicional quanto com o compensador neural.
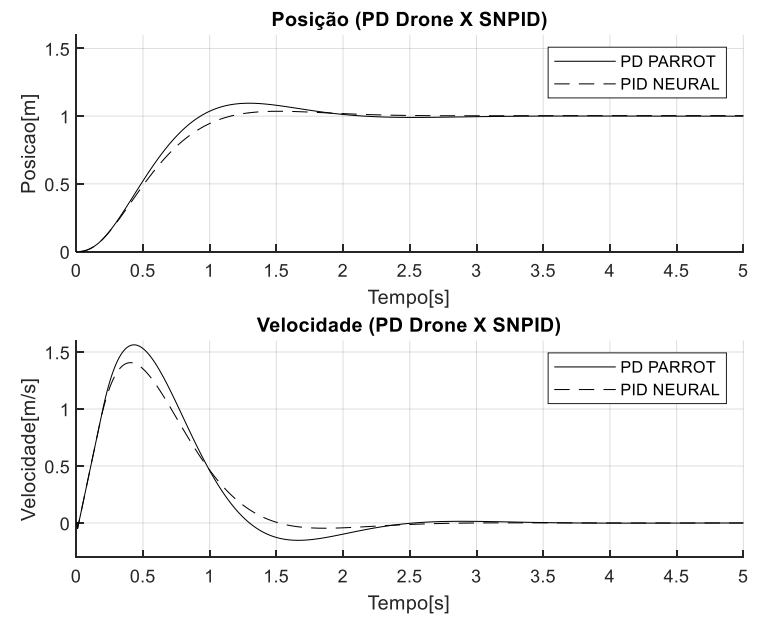

Fig. 6: Posição e Velocidade (PD Drone X PID Neural)

Na Fig. 6, o sistema compensador apresentou um pico menor estado de velocidade menor que o PD tradicional. No estado da posição houve uma redução do sobressinal do PID neural sobre o PD Parrot.

\subsection{Avaliação Comparativa dos Controladores PD e PID Neural via Esforço de Controle}


O esforço de controle é também apresentado e avaliado na Fig. 7. De acordo com esta figura, o controlador PD tradicional apresenta uma saturação entre 0 e 0.5 segundos e, por seguinte, um decaimento acentuado para depois retornar uma estabilização.

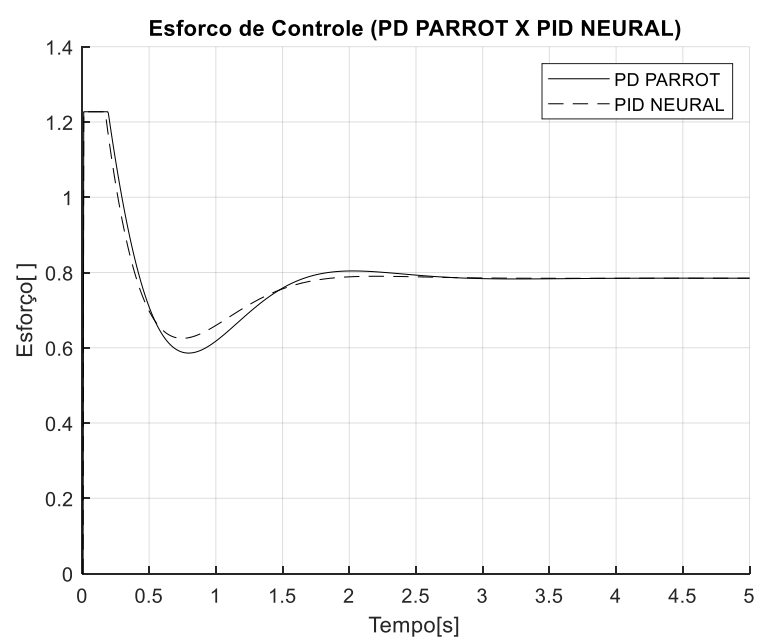

Fig. 7: Esforço de Controle (PD Drone X PID Neural)

O controlador PID Neural seguidor apresentou uma queda menor no esforço de controle em relação ao PD Parrot como também o retorno ao valor 0.8 .

\subsection{Sinal de Erro dos Controladores PD e PID Neural}

Na Fig. 8, o erro do controlador PD decaiu de forma menos intensa que o controlador PID neural. Contudo, ambos os controladores atingem o erro nulo ao mesmo tempo.

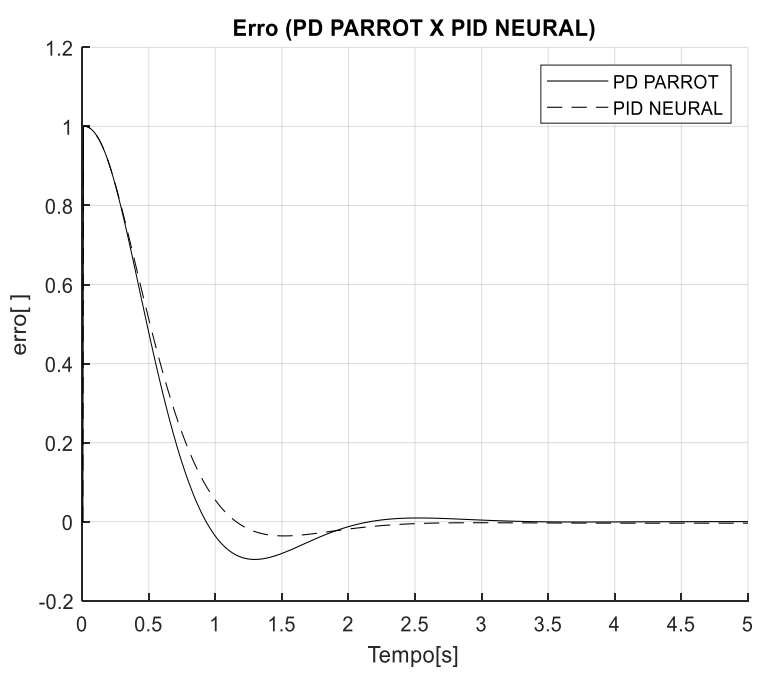

Fig. 8:Análise comparativa do erro (PD Drone X PID Neural)

\subsection{Rejeição às Perturbações}

Perturbações e variações ocorrem nos ambientes industriais em geral e produzem efeitos indesejáveis na performance e na estabilidade do controle de processos, a rejeição ao distúrbio e variações é uma das metas no controle de sistemas (Li, et al., 2016).

$$
\ddot{z}=-g+(\cos \phi \cos \theta) \frac{1}{m} U_{1}+0,2 * \text { rand, }
$$

Na Fig.9, um sinal de perturbação $20 \%$ do valor de referência é adicionado a dinâmica da planta, mostrada na Eq.15. Neste caso é possível perceber que o controlador PID Neural reagiu melhor a perturbação que o controlador PD Parrot.

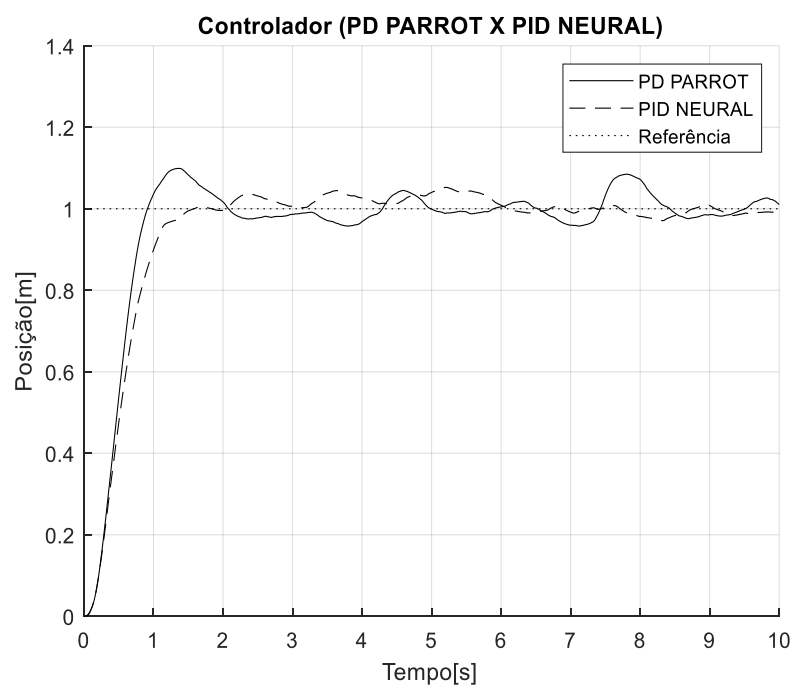

Fig. 9: Controladores PD Parrot e PID Neural com perturbação na dinâmica.

O sinal de erro dos controladores com perturbação ao degrau unitário é mostrado na Fig.10. Ambos foram sujeitos ao mesmo distúrbio e o controlador PID Neural ainda consegue se manter próximo de zero em comparação ao PD Parrot.

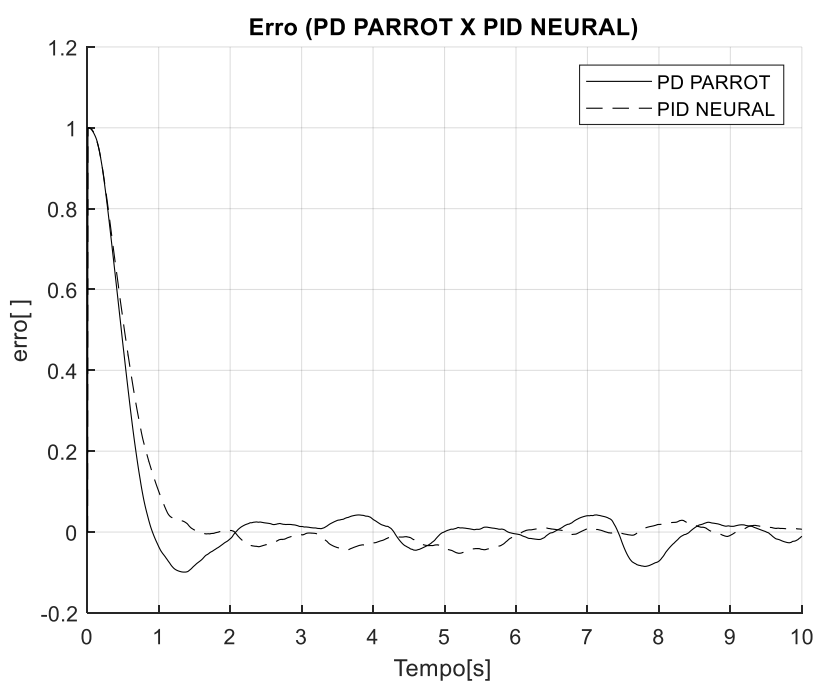

Fig. 10: Curva de erro dos controladores PD Parrot e PID Neural com perturbação na dinâmica. 


\section{CONCLUSÃO}

Neste artigo foi apresentado um sistema de compensação de controlador PID via Redes Neurais artificias para altitude de quadrimotor foi levantado o modelo matemático para a dinâmica de altura, apresentado a estrutura de um compensador neural (PID Neural e a Rede de ajuste de ganhos) e por fim resultados e simulações foram realizadas.

De acordo com os resultados e análise dos experimentos simulados, notou-se que o compensador neural consegue bons resultados em relação ao controlador PD tradicional e maior rejeição a perturbação. Portanto, as melhorias proporcionadas pelo PID Neural e a rede de ajuste de ganhos são superiores ao PD parrot.

\section{AGRADECIMENTOS}

Agradecemos à Universidade Federal do Maranhão pelo conhecimento técnicos/científicos e práticos para o desenvolvimento deste trabalho. Agradecemos também, à FAPEMA com o LCP (Laboratório de Controle de Processos) por fornecer o ambiente de trabalho e equipamentos para desenvolvimento deste projeto. Finalmente, à CAPES e CNPQ pelo financiamento desta pesquisa.

\section{REFERÊNCIAS}

Bouadallar, S., Murrieri, P. \& Siegwart, R., 2006. Design and Control of an Indoor Micro Quadrotor.

Bresciani, T., 2008. Modelling, Identification and Control of Quadrotor Helicopter, Lund: Lund University.

Fonseca Neto, J. V., Franca Coelho, B. \& Rego Moraes, P., 2018. Sintonia Online De Controladores Pid Sintonizado Por Regras Fuzzy Em Sistemas Industriais Com Aplicação Em Processo Operacional De Viradores De Vagões. Curitiba, s.n.

Haykin, S., 2008. Neural Networks and Learning Machines. 3 ed. New Jersey: Prentice Hall.

Jinkun, L., 2017. Inteligent Control Design and MATLAB Simulation. $1^{\mathrm{a}}$ ed. Beijing: Springer.

Jun, L. \& Yuntang, L., 2011. Dynamic analysis and PID control for a quadrotor. Beijing, China, s.n.

Li, S., Yang, J., Chen, W.-H. \& Chen, X., 2016. Disturbance observer-based control: methods and applications. $1^{\mathrm{a}} \mathrm{ed}$. s.l.:CRC Press.

Liu, J., 2016. On a Method of Single Neural PID Feedback Compensation Control.. Harbin, IEEE.

Liu, J., 2017. Inteligent Control Design and Matlab Simulation. $1^{\text {a }}$ ed. Beijing: Springer.

MATHWORS, 2018. Parrot Mini Drones Support from Simulink.

[Online]

Available at: https://www.mathworks.com/hardwaresupport/parrot-minidrones.html [Acesso em 25 Abril 2018].

MORADI, M. \& JOHNSON, M., 2005. PID Control New Identification. London: Springer.

Moura, J. P., Franca Pinheiro, B. \& Fonseca Neto, J. V., 2018. Controlador pid para o controle de vazão de sólidos em sistemas industriais com aplicação em viradores de vagões. Curitiba, s.n.
Necdet, O., Mert, O. \& Mehmet, E., 2015. Feedback control strategies for quadrotor-type aerial robots: a survey. Transactions of the Institute of Measurement and Control, 38(SAGE), pp. 529-554.

Quan, H., 2017. Introduction to Multicopter Design and Control. Singapore: Springer .

Rong, X. \& Umit, O., 2006. Sliding Mode Control of a Quadrotor Helicopter. San Diego, s.n.

Shifei, D. \& Hui, L., 2011. Evolutionary artificial neural networks: a review.. Artificial Inteligence Rev, 17 junho, pp. 251-260.

Vijay, K., Robert, M. \& Peter, C., 2012. Multirotor Aerial Vehicles: Modeling, Esimation and Control of Quadrotor. IEEE ROBOTICS \& AUTOMATION MAGAZINE, 21-32 Agosto, p. 1070.

Xiwen, L., 2008. Single Neuron Self-tuning PID Control for Welding Molten Pool Depth. Chongqinq, IEEE.

Yibo, L. \& Shuxi, S., 2012. A Survey of Control Algorithms for Quadrotor Unmanned Helicopter. Nanjing, s.n. 